
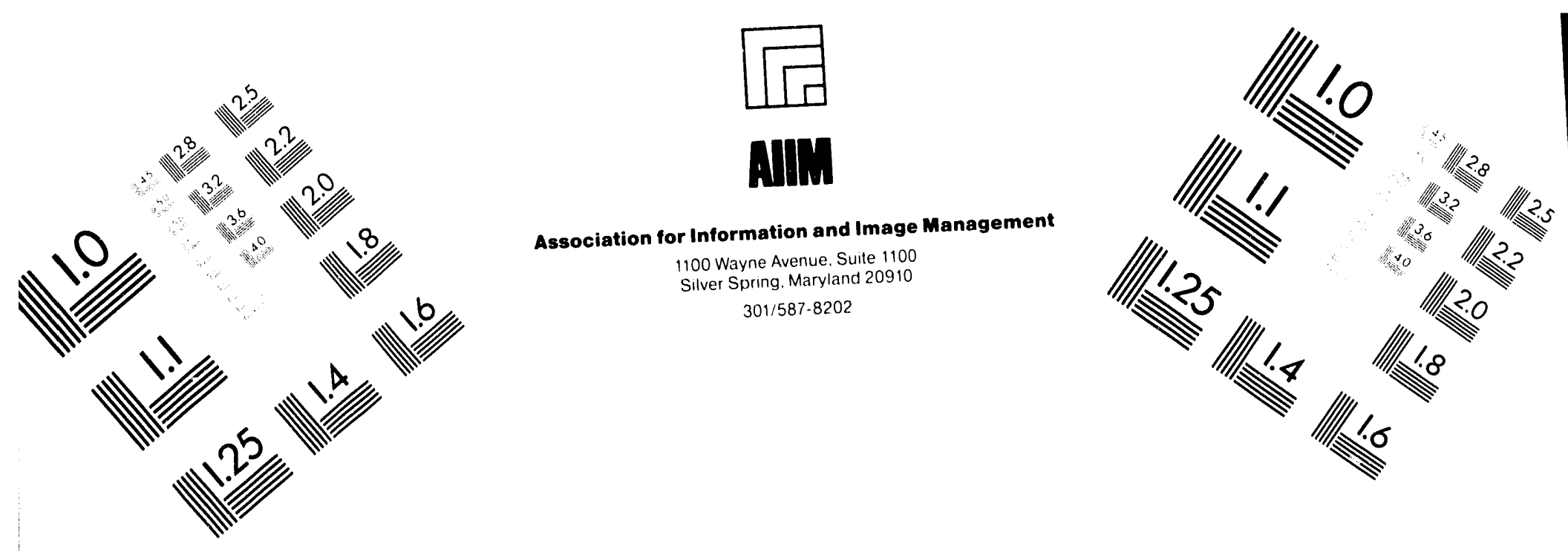

Centimeter

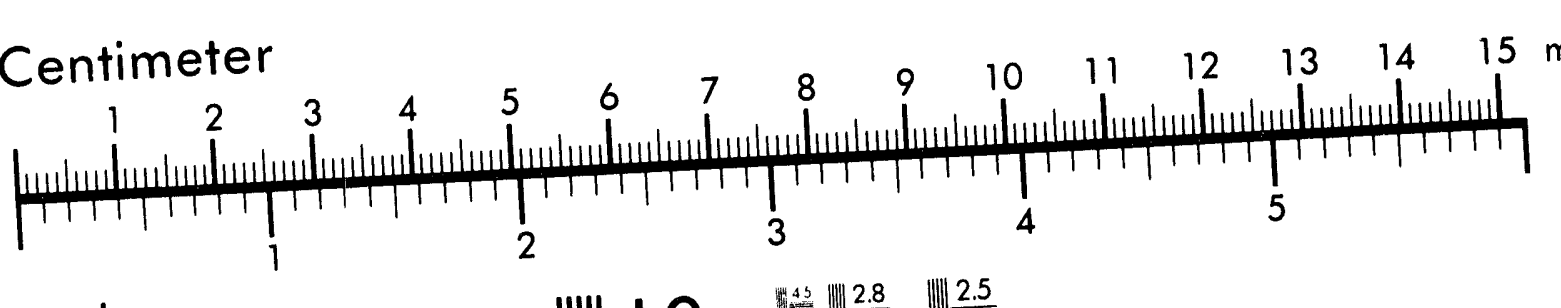

Inches
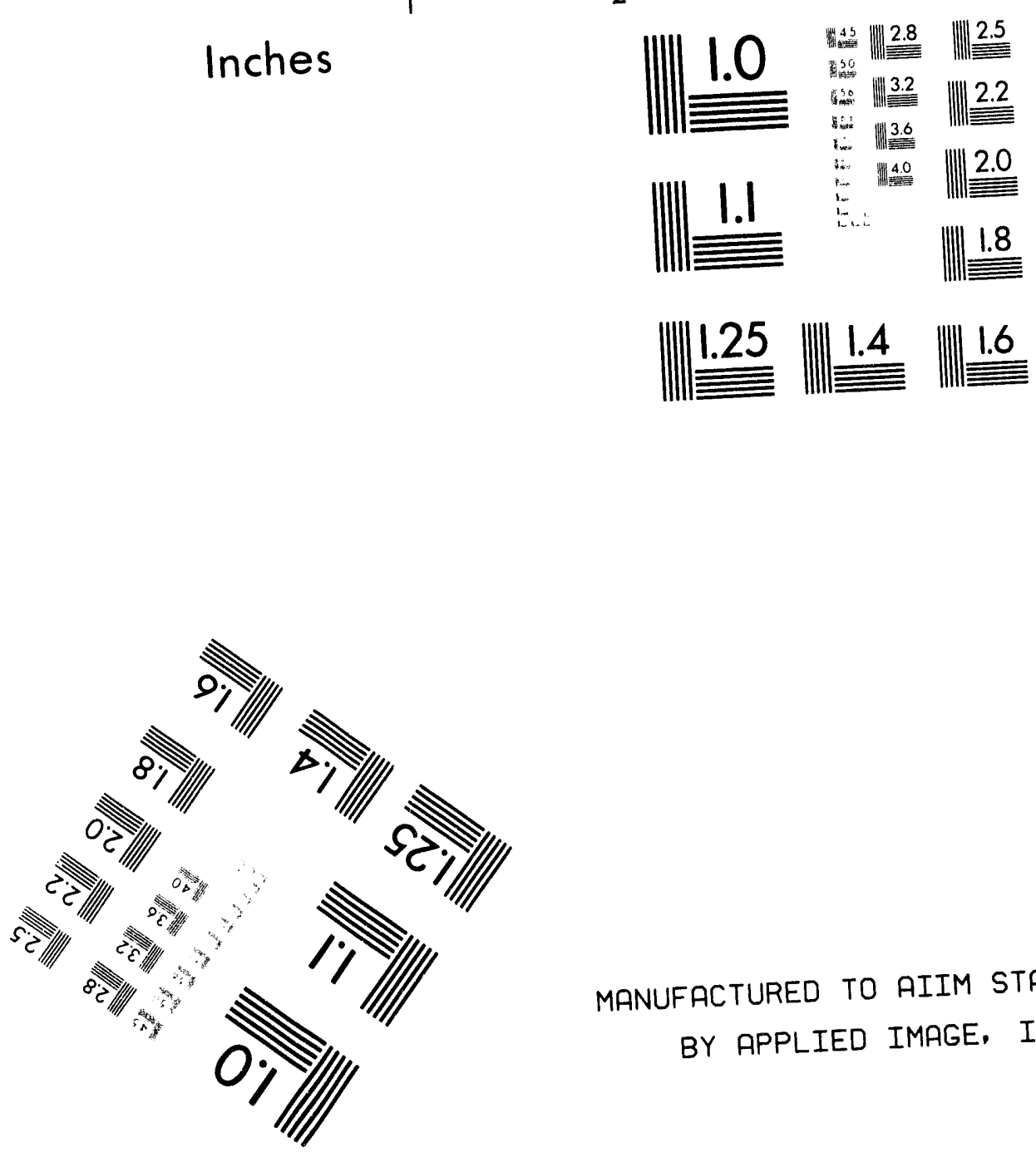

MANUFACTURED TO AIIM STANDARDS

BY APPLIED IMAGE, INC.

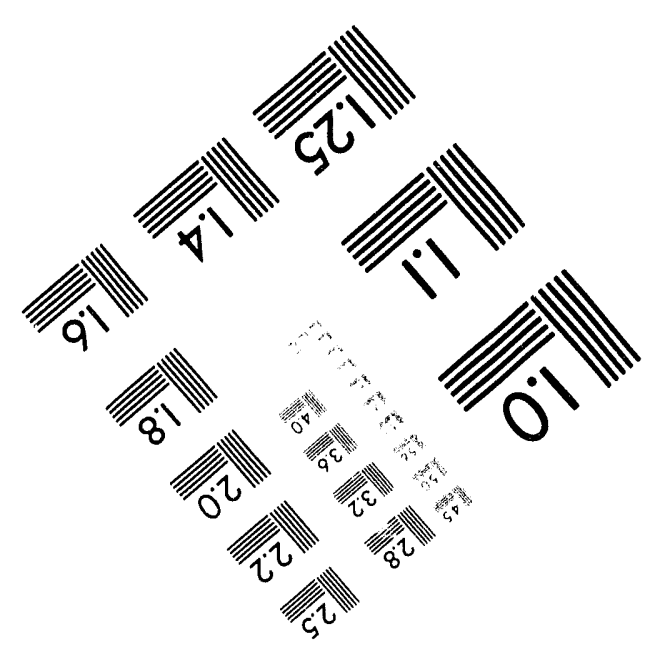



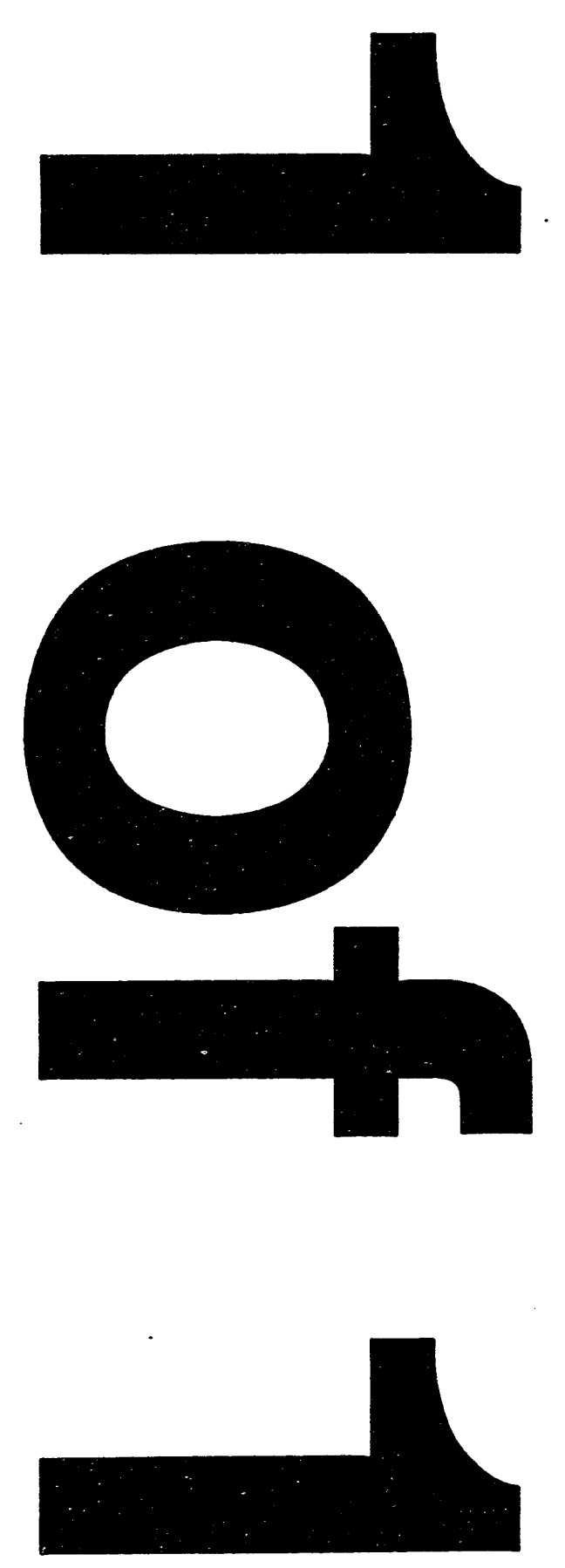
$1 / 2 / 20.94980$

UCRL-ID-116758

\section{U-Turn Alternative to the Large Aperture Switch}

Charles S. Vann

March 9, 1994

This is an informal report intended primarily for internal or limited external diveribution. The epialons and conclualons ctated are those of the author and may or ming not be thowe of the Laboratory.

Work performed under the awopices of the U.S. Department of Energy by the Lawrence Ltvermare National Laboratary under Contract W-7405-Eng-48.

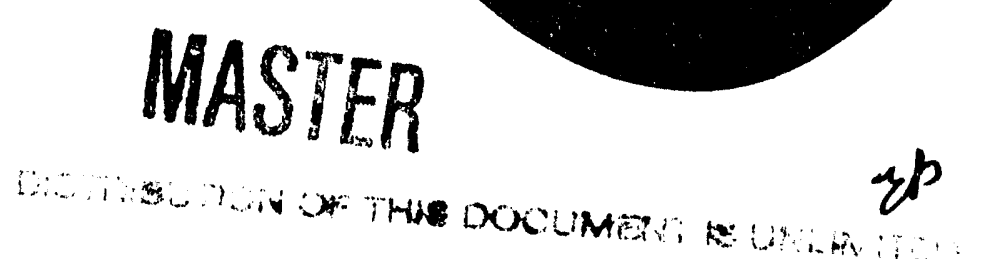




\section{DISCLAMMER}

This document was prepared as an account of work sponsored by an agency of the United States Government. Neither the United States Covernment nor the University of California nor any of their employees, makes any warranty, express or implied, or assumes any legal liability or responsibility for the accuracy, completeness, or usefulness of any information, apparatus, product, or process disclosed, or represents that its use would not infringe privately owned rights. Reference herein to any specific commercial product, process, or service by trade name, trademark, manufactures, or otherwise, does not necessarlly constitute or imply its endorsement, recommendation, or favoring by the United States Government or the University of California. The views and opinions of authors expressed herein do not necessarily state or reflect those of the United States Government or the University of California, and shall not be used for advertising or product endorsement purposes.

This report has been reproduced directly from the best available copy.

Available to DOE and DOE contractors from the Office of Scientific and Technical Information P.O. Box 62, Oak Ridge, TN 37831

Prices available from (615) 576-8401, FTS 626-8401

Available to the public from the

National Technical Information Service

U.S. Department of Commence 5285 Port Royal Rd. Springfield, VA 22161 


\subsection{U-turn Alternative to the Large Aperture Switch}

The primary alternative laser architecture is the U-turn design (see Figure 1). The U-turn has significantly different cost and performance risks than the full-aperture switch, which makes it a highly desirable alternative. The Uturn was conceived at LLNL in 1992. A similar concept, the L-turn had already been discovered by the French at CEL-V. Both concepts are based on the multipass glass amplifier design, but the full-aperture Pockels cell and polarizer are replaced with smaller and less expensive optics. Eliminating the large switch and polarizer not only reduces component costs, it also provides options for shortening the laser which, in turn, could reduce the size and cost of the laser building. Efficient use of the amplifier aperture (small vignetting allowance) requires that the U-turn have a long transport spatial filter; however, this is not a disadvantage if a long spatial filter is already required for image relaying to the frequency converter. Given a long spatial filter, the U-turn is potentially more efficient because losses in the switch and polarizer are avoided.

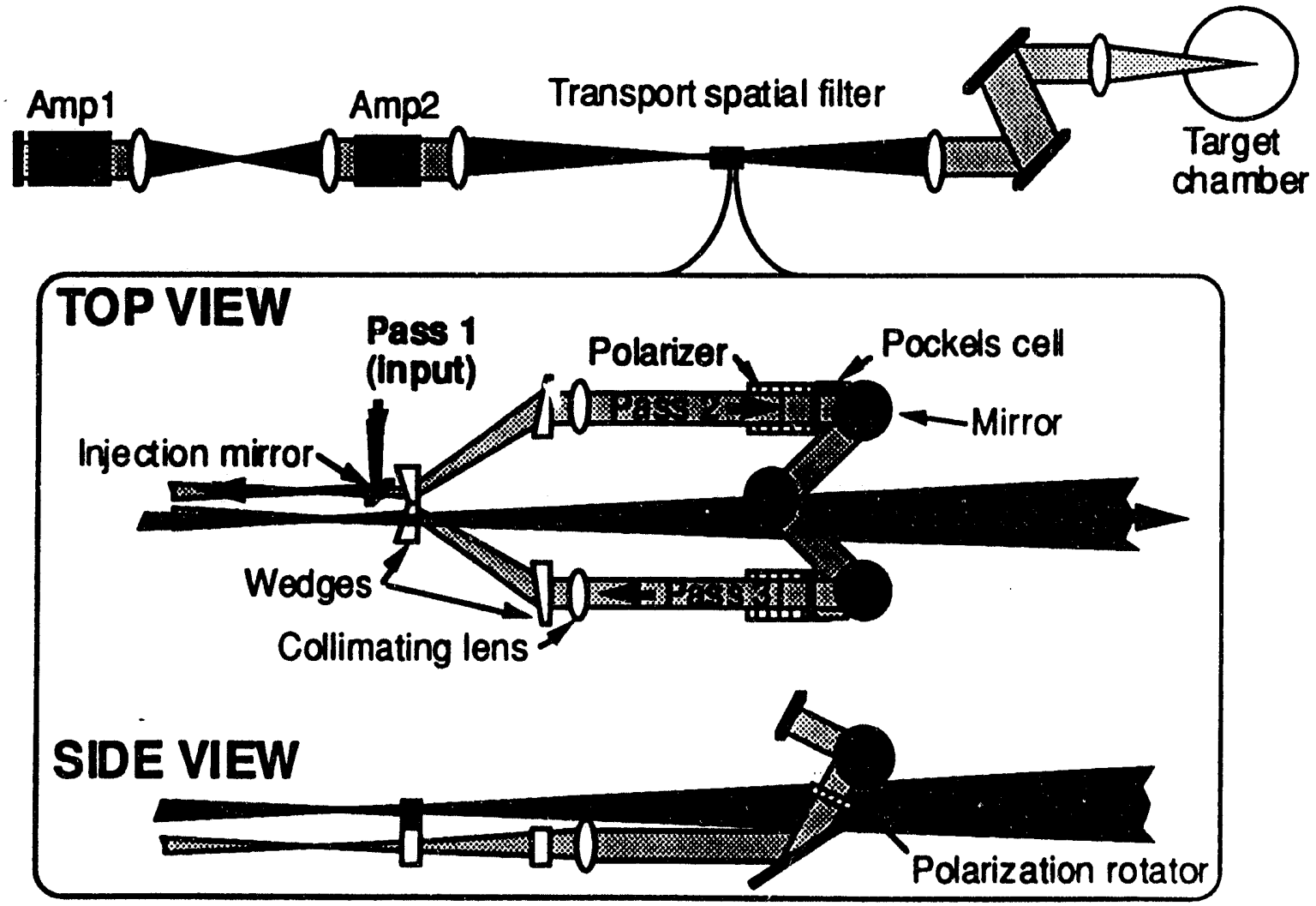

Figure 1, Schematic of the U-turn

The L-turn is the baseline for the French Megajoule laser, the corollary to NIF. A small-scale optical prototype of the L-turn is already operational at Limeil. Fabricating the U-turn is planned this year. Both the $\mathrm{L}$ and U-turn are scheduled for testing in Beamlet in early FY 1995. If the Beamlet tests are 
successful, the U-turn could be incorporated into the preliminary NIF design during Title I.

\subsection{Description of the U-turn}

The U-turn is an optical device which enables four passes through an amplifier without the need for a large aperture switch. The U-turn consists of small optics located inside the output spatial filter which pick-off the beam after pass two through the amplifier and turn it around to make pass three (see Figure 1). The U-turn hardware consists of wedges to divert the beam, lenses to collimate/focus and properly relay the beam, a Pockels cell and polarizers for ASE isolation, and three mirrors to invert the beam.

The wedges divert the beam off-axis permitting other components to be included in the U-turn without interfering with the final pass. Wedges are used rather than mirrors because of their higher damage threshold and are paired to cancel their aberrations. The lenses collimate and properly relay the beam at a large enough beam size to avoid optical damage to the U-turn components, probably around $5 \times 5 \mathrm{~cm}$.

The fintolarizer reflects only the light with the desired polarization and passe. in themainder to a dump. The polarizati thotator changes the beam polary.

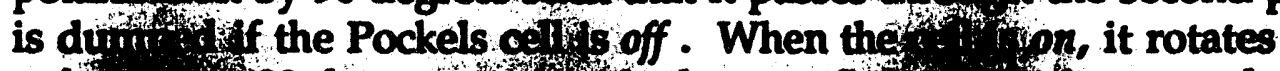
polat, in 90 degrees so the beam reflection the second polatizer and exituthe U-turn.

Three mirrors, located between the Pockels cell and the polarization rotator, invert the beam image in both dimensions like a corner cube. In the baseline, beam pointing and focusing errors caused by vibrations, thermal drift, and pump induced steering add with the number of passes. By inverting the beam in the U-turn, all the pointing errors accumulated on passes one and two are subtracted (rather than compounded) on passes three and four. This does not apply to the focusing errors, but those can be corrected with the wavefront correction system. In this sense, the cavity is self-stabilizing. The three turning mirrors are positioned like the faces of a corner cube such that the beam leaves the three-mirror combination parallel but opposite in direction from the way it enters. The beam polarization is unchanged.

The exit optics are exactly the same as the entrance optics with a second lens to re-focus the beam and two more wedges to redirect the beam onto pass three through the amplifiers. The second lens and one of the mirrors are motor controlled to point and center the beam through pass three. An insertable polarization rotator permits beam alignment without firing the Pockểs cell. 
Other U-turn designs exist. Instead of inverting the beam, the U-turn could rotate it 90 degrees. Wavefront distortions also increase exponentially with the number of cavity passes, and most beam distortion is horizontal. Rotating the beam 90 degrees after pass two, exchanges the distorted horizontal with the less aberrated vertical dimension. Thus, the output wavefront distortion becomes symmetric in both axes and lower in amplitude, as if there were only two passes. However, this arrangement does not correct alignment errors.

In contrast, the French L-turn does not invert or rotate the beam. The L-turn picks-off pass two but does not optically separate its path from pass three. The two passes share a pick-off mirror, collimating lens, Pockels cell, polarizer, and cavity mirror. The result is fewer optics and potentially less vignetting between pass two and three. On the downside, the system does not reduce alignment errors or wavefront aberrations because the beam cannot be manipulated without separating the passes. Also, the L-turn optics are used in double pass, which increases the energy in the turn. Thus, the L-turn optics will likely be larger than U-turn optics to reduce risk of damage.

\subsection{Description of the U-turn laser}

An important benefit of the U-turn is that in shortens the laser which reduces the size of the building needed to house it. The laser is shorter for several reasons. An obvious reduction in beam path length comes from elimination of space in the cavity previously occupied by the switch and polarizer. Since the length of the cavity determines the length of the spatial filter, a corresponding reduction in spatial filter length is realized.

Furthermore, the beam leaves the U-turn cavity on axis, eliminating the two level characteristic of the baseline. This eliminates mirror LM3 (elbow mirror) and the beam path length associated with it. Without the polarizer, there is no need for a separate booster amplifier, so those slabs are incorporated into amplifier two (see Figure 2).

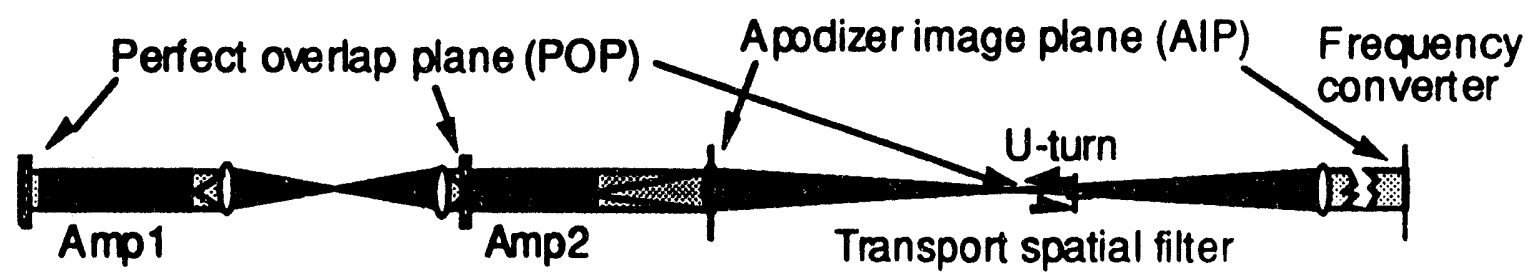

Figure 2, Proposed U-turn laser configuration for NIF

The laser cavity mirrors are located in the U-turn and at the opposite end of the amplifier one. Unlike the baseline, this cavity arrangement has two optical relay systems: the cavity spatial filter and another comprised of the 
input lens to the transport spatial filter and the U-turn lenses. This means the cavity spatial filter does not have to relay the entire cavity resulting in a much shorter length. The beam still perfectly overlaps on the cavity mirrors for all four passes, but there is an additional perfect overlap plane (POP) between them (see Figure 2).

A shorter transport spatial filter length is also realized with this architecture. This is accomplished by optically placing the apodizer image plane (AIP) on its input lens during pass four. This reduces the subsequent relay distance to the frequency converter which in turn reduces the required length of this spatial filter (see Figure 2). Also, from a damage point of view, this is the best place for the AIP. On pass four, this lens sees the highest B integral in the system. At the relay plane of the apodizer, beam ripple is minimum which will improve the survivability of this lens by lowering the peak fluence.

\subsection{Advantages of the U-turn laser}

\subsection{1 Lower Cost per Beamline}

Eliminates the large switch: The U-turn is about one fifth the cost of the fullaperture switch and polarizer.

Reduces the size of front-end: In the U-turn laser, the beam passes through all the slabs four times. In contrast, the beam passes only two times through the booster amplifier in the baseline. Since the U-turn laser has more slab passes for an equivalent number of slabs, the injection energy required is lower. Additional reduction in required input energy is achieved by the elimination of losses in the switch and polarizer. Since the pre-amp size is set by the energy needed at longer pulses, better performance at longer pulse lengths reduces the injection requirement further (discussed below). This reduction in injection energy corresponds to a smaller front-end which simplifies and lowers its cost.

Eliminates full-aperture optics: LM3 (elbow mirror) is eliminated, and LM2 (a full-aperture cavity mirror) is replaced with a much smaller U-turn mirror.

Eliminates the beam dump: The beam dump absorbs energy not reflected by the switch/polarizer. This is not required with the U-turn.

May eliminate the need for Noble gas box: The distance between the output spatial filter and the frequency converter may be less than $45 \mathrm{~m}$ (discussed below). This would be below the distance in which Stimulated Raman Scattering (SRS) and excessive B integral accumulation in the beam path become an issue, thus, eliminating the need for the noble gas box and its window. 
5.3.6.8.5.3.2. Lowers technical risks: Making a large aperture switch and polarizer has higher fabrication risk than making the U-turn. Also, there is operational risk with the switch. If it fails, pass five could damage the laser. Operational risks associated with the U-turn need further evaluation.

5.3.6.8.5.3.3. Reduces the building size: Beam pointing errors caused by vibrations, thermal drift, pump induced beam steering, etc. increase exponentially with the number of passes in the cavity. Consequently, the structural stability requirements for the laser cavity are difficult and make impractical the stacking of laser bays to reduce building space. The U-turn relaxes this stability criteria by making the laser self-compensating with respect to pointing errors. This is accomplished by inverting the beam inside the U-turn such that the pointing errors accumulated on pass one and two are subtracted, rather than compounded, during pass three and four. With the laser self-stabilizing, the option of stacking two laser bays becomes plausible (see Figure 3).

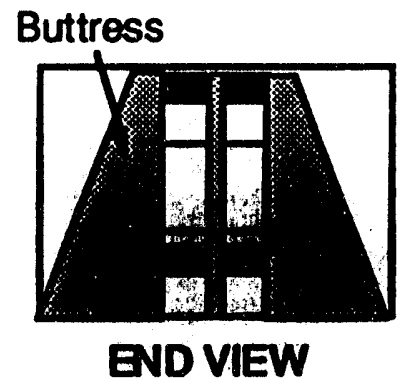

\section{SIDE VIEW}

END VIEW

Figure 3, The self-stabilizing feature of the U-turn permits stacking of laser bays

5.3.6.8.5.3.4. Reduces transport path length: Given that the amplifiers can be stacked, the beam path length can be shortened. A building layout is proposed that has four laser bays paired high and low. Two chambers are symmetrically located between the bays such that the beams can be switched from one chamber to another without changing the path length (see Figure 4). With this layout the height of the laser bays can be selected such that the beams transport to the target chamber with minimal vertical path length reducing the path by several meters. With twice as many bays, the width of each bay is halved. Less width results in shorter beam paths to crossover from one chamber to another. The beams are bundled four high and six wide, i.e. two bundles side by side. Each bundle maps to a separate octasphere by the shortest route (see Figure 4). This results in an arrangement where the chambers are closer together with beam path lengths reduced accordingly. The path length from the transport spatial filter to the frequency conversion 
crystal is expected to be less than 45 meters. The pclarization flip, same number of turning mirrors, and vertical target chamber are maintained.

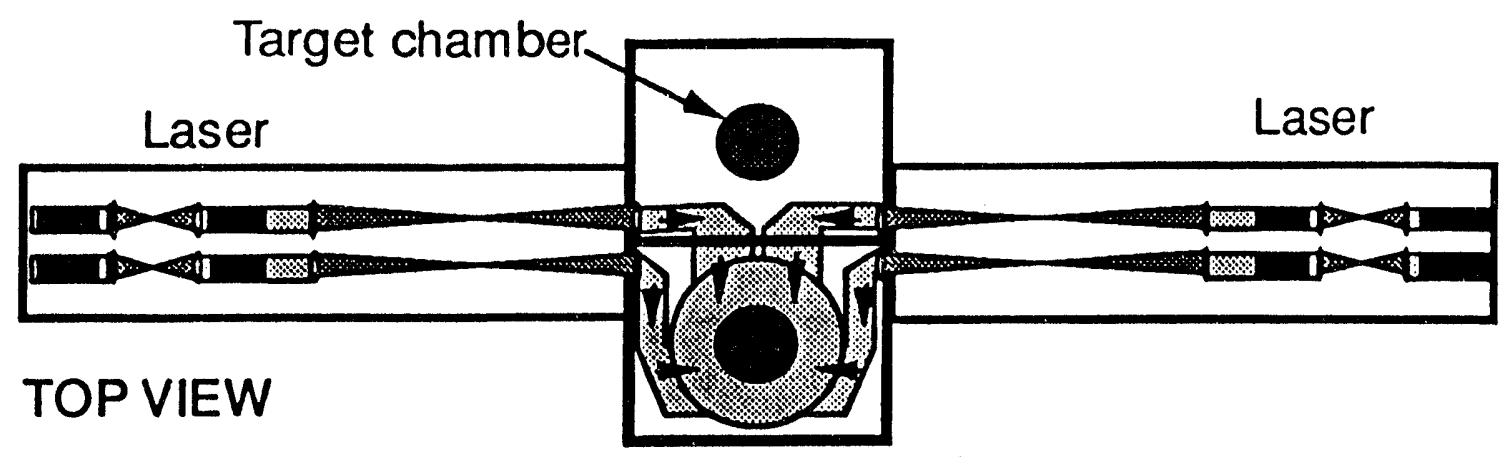

Figure 4, Plan view of a U-turn laser with stacked laser bays

5.3.6.8.5.3.5. Reduces building costs: Since the laser and beam transport are shorter, the building housing the U-turn laser can be smaller in area although it will probably be taller (see Figure 5).

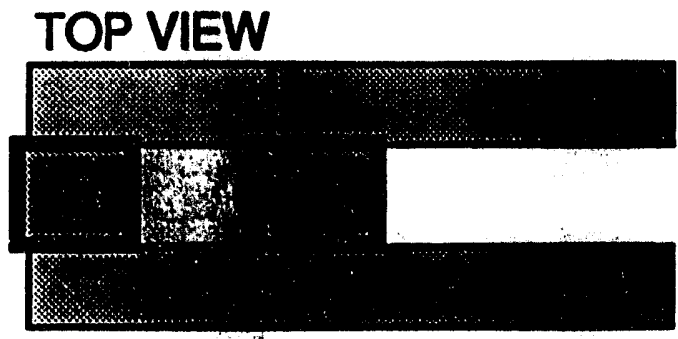

Baseline building

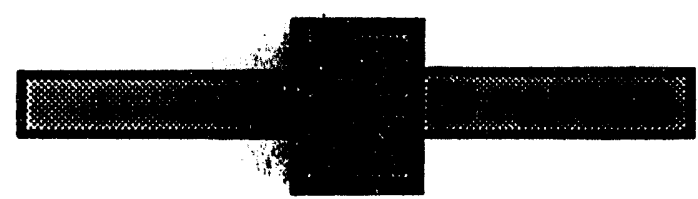

U-tum building

Figure 5, Comparison of the Baseline and a U-turn buildings

5.3.6.8.5.3.6. Extracts more energy per beam: The U-turn laser is more efficient than the baseline because the losses and $B$ integral associated with the switch and polarizer are avoided. Also, losses to vignetting are less even with the reductions in the length of the transport spatial filter. This is primarily because vignetting in the baseline must be greater in the vertical dimension to prevent damage to the cavity beam dump. The U-turn has no beam dumps. In the horizontal dimension, vignetting for both the baseline and the U-turn is dictated by the minimum allowable spacing between cavity pinholes $(\sim 1 \mathrm{~cm})$. When the minimum spacing is optically magnified to the transport spatial filter, it subtends an area larger than the size of the first wedge in the U-turn or the injection mirror.

With better extraction efficiency and less vignetting, preliminary analysis show the U-turn has greater 1w output than the baseline (Figure 6). At the 
third harmonic, the relatively low damage threshold of the tripler limits the U-turn performance to that of the baseline. Given that more energy cannot be extracted through the tripler, the better efficiency of the U-turn could be used to lower cost instead. For example, the same amount of energy output could be provided with less laser glass reducing costs substantially.

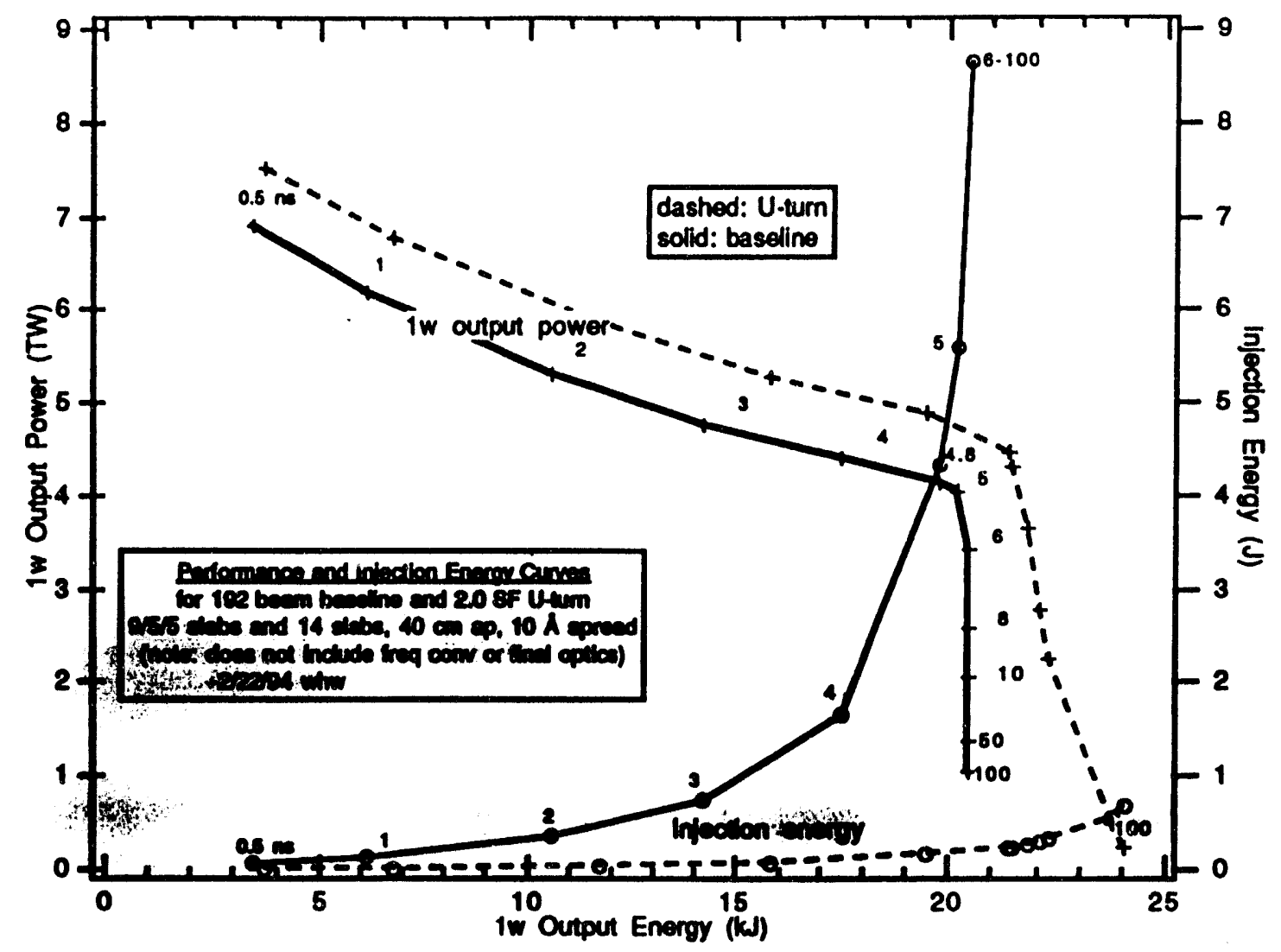

Figure 6, Output energy/power versus pulse length

\subsection{Disadvantages of the U-turn Laser Architecture}

As a relatively new concept, very little effort has been expended on evaluating the U-turn. Consequently, there may be problems with the U-turn which we are not yet aware. For example, there are questions about parasitics, beam isolation, and homogeneity of the Pockels cell. These questions should be addressed with further evaluation, propagation analysis and subsequent testing of the hardware.

\subsection{Testing the U-turn on Beamlet}

As a potential carididate for NIF, the U-turn requires validation with fullscale hardware. This is planned in FY 1994. The hardware will be modular 

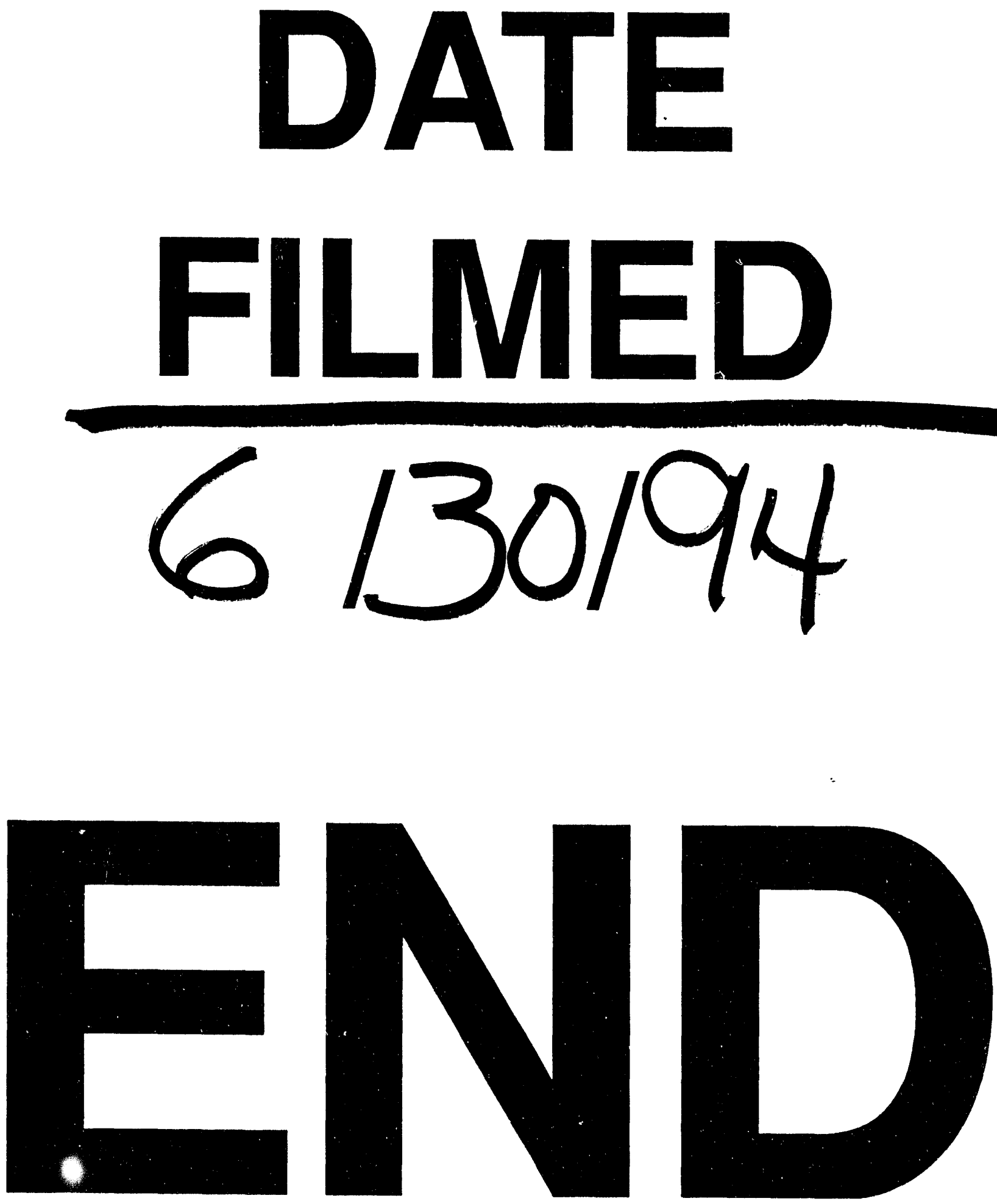
